\title{
Enhanced Inter Cluster Data Aggregation based RN- LEACH Protocol using Hybrid PSO-GA
}

\author{
Rajwinder Kaur \\ M-tech Student \\ DAV Institute of Engineering \&Technology \\ Jalandhar, India.
}

\author{
Shaveta Angurala \\ Assistant Professor \\ DAV Institute of Engineering \&Technology \\ Jalandhar, India
}

\begin{abstract}
Wireless sensor networks consist of battery driven devices that contain limited energy resources. Due to the placement of sensor nodes in a hostile environment it is very difficult to recharge the batteries of nodes .So limited energy resource affects the lifetime of the network. Number of protocols has been proposed to reduce the dissipation of energy. This paper has proposed a novel rendezvous nodes based LEACH (RNLEACH) by using the hybrid PSO-GA based inter cluster data aggregation. The hybrid PSO-GA based technique has improved the path selection between the sink and cluster heads to reduce the energy consumption of cluster heads in an optimistic manner. The results have clearly shown that the proposed technique outperforms over the available methods.
\end{abstract}

\section{Keywords}

Inter cluster data aggregation, Clustering, Mobility, Rendezvous node (RN), Energy efficiency, Network lifetime.

\section{INTRODUCTION}

Recent advancements in large scale integration and wireless communication technologies have enabled the development of small size, low cost, and multi-functional devices known as sensor nodes [2].Deployment of the large number of sensor nodes is done either inside the phenomenon or very close to it[3]. Sensor nodes deployed over a geographical area for monitoring physical phenomena like temperature, humidity, vibrations, seismic events, and so on[1]. Variety of sensing capabilities results in profusion of application areas [4]. Sensor networks are mostly used in, low bandwidth and delay tolerant applications ranging from civil and military to environmental and health care monitoring [6]. In various applications sensor network is organized in a random manner ( in a disaster management application nodes are dropped with the help of aero plane)[5]. Sensor nodes are energy constraint so to improve the lifetime of the network numbers of innovative techniques are required [7].These techniques will help to reduce the energy consumption of network. There are number of communication protocols that have significant impact on the overall network lifetime[9]. LEACH is the first protocol of hierarchical routings which proposed data fusion. This protocol has important significance in clustering routing protocols. Many hierarchical routing protocols are improved ones based on LEACH protocol [8]. LEACH is a mature clustering based protocol which reduces the energy dissipation by dividing the network into clusters to restrict direct communication between nodes and base station. Although LEACH is better than conventional routing protocols as the idea of rounds distributes transmissions around all nodes, and also data aggregation by cluster-heads reduces energy dissipation of the network but LEACH still ignores the residual energy at each node during the cluster-heads selection stage [10].So a rendezvous-based data collection approach that explores the controlled mobility of sink node and the capability of in-network data caching is another approach to improve the lifetime of the network[11]. In this approach rendezvous points are made near the trajectory of a mobile sink (MS). A mobile sink (MS) is an alternate way to reduce energy consumption [12]. The use of rendezvous points helps the cluster heads to collect a large volume of data at a time without traveling a long distance which achieves high data bandwidth and low communication delay at the same time and increases the lifetime of the network [13].

In the present study improvement will be done in the rendezvous nodes based LEACH by using the hybrid PSO-GA based routing algorithm. This algorithm provides the shortest path between the cluster heads to base station. Comparison of the rendezvous nodes based LEACH and Hybrid PSO-GA is done on the basis of various parameters.

The remainder of the paper is organized as follows: Section II presents the related work. Section III presents the proposed algorithm and section IV describes the experimental results. Finally, section V concludes the paper.

\section{RELATED WORK}

In [14] importance of wireless sensor networks is clearly shown. Various application areas in which the sensor networks used are properly elaborated. Architecture of the sensor networks and the various factors that affect the network design are shown. Discussion about various layers of network and protocols that are used for communication are also described. Various research issues are discussed in detail. There are number of routing protocols that are made for the wireless sensor network to increase the lifetime of the network. Recently used routing protocols [15] for the sensor networks are discussed and also the categories of various approaches are explored. Data centric, hierarchical and location based categories are the main categories that are discussed properly. Energy consumption of nodes is the major issue in all he sensor networks. So [16] provides a routing protocol LEACH that is a clustering based energy efficient protocol used for the mobile sensor networks. Working of this protocol is done in rounds. By introducing the various features for the mobile nodes it reduces the dissipation of energy in every round [17] provides a clustering based approach that is used to increase the energy efficiency of sensor network. This paper improves a LEACH protocol in which clusters are made periodically based on the distance and the remaining energy. All the nodes will send the data in the allotted time slot and keep their receivers off in rest of the time to save the energy. Proposed method MOD LEACH provides better energy efficiency than the LEACH protocol.[18] proposed a algorithm (ES-WCA) for mobile wireless senor network by using five metrices. This protocol is introduced to prolong the battery life of the each sensor node. To increase the lifetime of the network [19] provides a genetic algorithm based approach (GABEEC).Genetic algorithm is used to increase the lifetime of the network and in this proposed method clusters are made same like the LEACH protocol.[20] presents a clustering protocol based on PSO to improve the energy efficiency of nodes. Fitness function is optimized in the proposed method for clustering. In [21] survey of recent progress mobile wireless sensor networks and work is 
compared in this field in terms of their models and mobility management methodologies. The discussion includes three aspects. Firstly, we discuss mobility management of mobile sensors for the purposes of forming a better WSN, enhancing network coverage and connectivity, and relocating some sensors. Secondly it introduces path-planning methods to relay data between isolated sensors and to extend the lifetime of the network. Various applications of sensor network are also discussed.

\section{PROPOSED ALGORITHM}

This section provides the flow chart and steps required for successfully accomplishing the objectives of the paper.

1. Deployment of nodes in the network region randomly.

2. Apply the threshold function of LEACH protocol to elect the cluster heads.

3. Associate the member nodes to the cluster heads according to the distance between the normal nodes and cluster heads.

4. Position of rendezvous nodes is evaluated on the basis width and position of nodes in the $y$ dimensions

5. Apply the PSO-GA algorithm to find the shortest path from cluster heads to base station

6. Evaluate the consumption of energy after sending the data to the base station

7. Count the number of dead nodes. Nodes becomes dead when their energy is zero and they cannot further send the data.

8. If all the nodes become dead then evaluate the lifetime of the network.

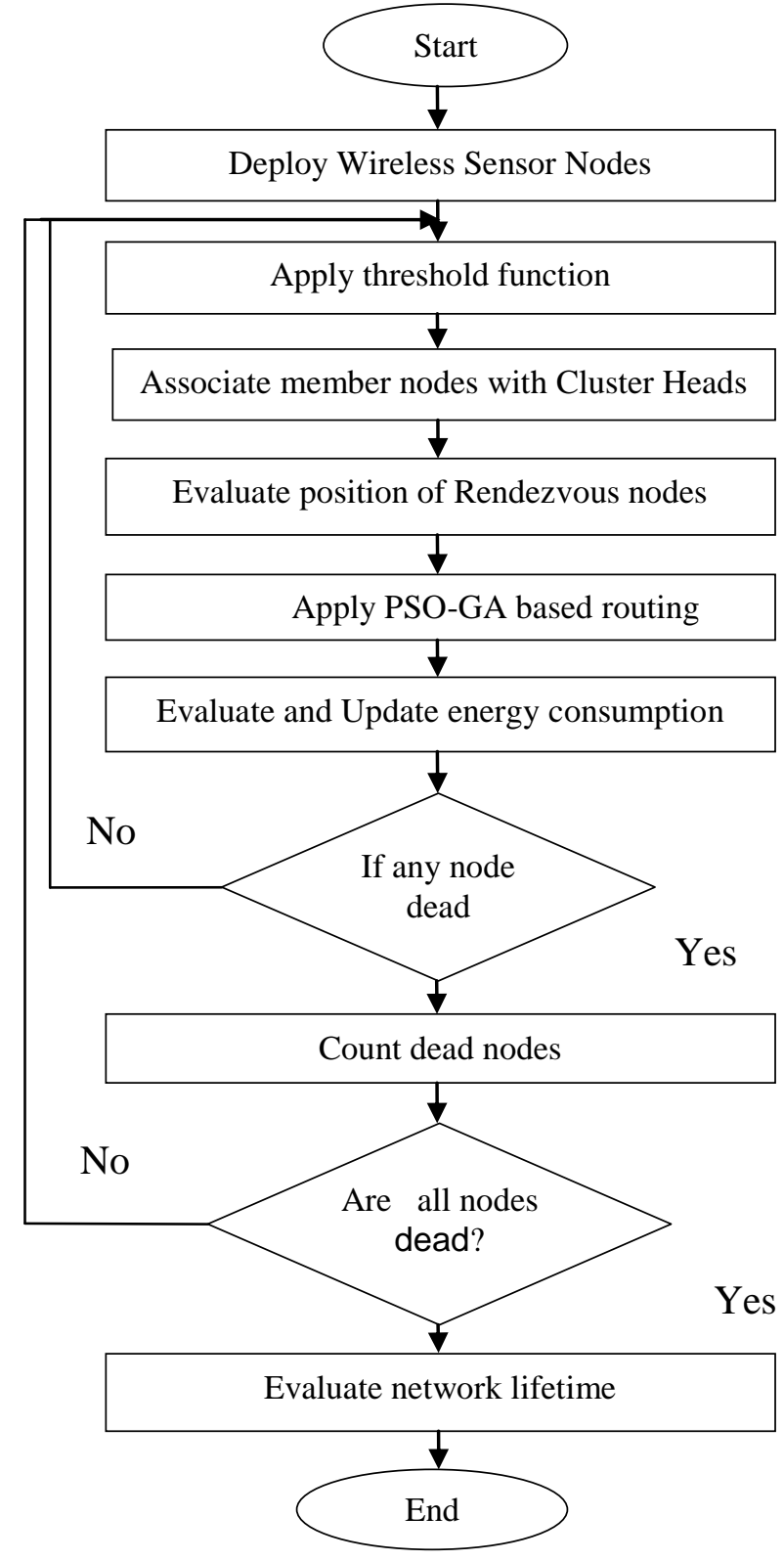

Flow Chart Of Proposed Algorithm

\section{EXPERIMENTAL RESULTS}

To evaluate the performance of PSO-GA algorithm simulation is done in MATLAB. Comparison of existing technique (rendezvous nodes based) LEACH is done with the proposed technique (Hybrid PSO-GA) by taking different number of nodes.

\subsection{Comparison On The Basis Of First Node Dead Time}

Table 1. shows the comparison of the RN-LEACH and the Hybrid PSO-GA on the basis of first node dead time. In the table we can see that in Hybrid technique dead time of first node occur later than RN-LEACH. 
Table 1. First Node Dead Time Analysis

\begin{tabular}{|c|c|c|}
\hline No. of nodes & RN-LEACH & Hybrid PSO-GA \\
\hline 40 & 951 & 876 \\
\hline 60 & 869 & 897 \\
\hline 80 & 831 & 1025 \\
\hline 100 & 857 & 1083 \\
\hline 120 & 876 & 924 \\
\hline 140 & 871 & 1014 \\
\hline 160 & 880 & 988 \\
\hline 180 & 1001 & 1156 \\
\hline 200 & 971 & 1080 \\
\hline 220 & 920 & 1068 \\
\hline
\end{tabular}

Fig 1. is showing graphical representation of RN-LEACH and Hybrid PSO-GA technique. X-axis is representing the number of nodes and $\mathrm{Y}$-axis is representing the rounds.

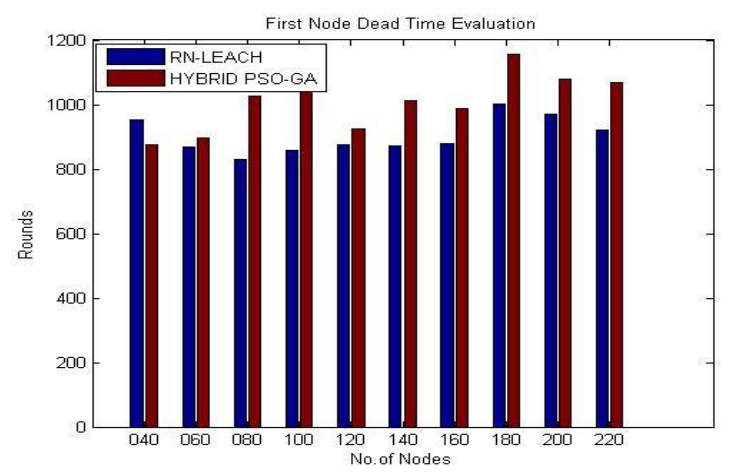

Figure 1. Graph when first node dies in RN-LEACH and Hybrid PSO-GA.

\subsection{Comparison on the basis of Tenth node dead time}

Table 2 shows the comparison of RN-LEACH and the Hybrid PSO-GA on the basis of tenth node dead time. In the table it is clearly shown that proposed algorithm performs better than the existing technique.

Table 2. Tenth Node Dead Time Analysis

\begin{tabular}{|c|c|c|}
\hline No. of nodes & RN-LEACH & PSO-GA \\
\hline 40 & 1005 & 1286 \\
\hline 60 & 1021 & 1313 \\
\hline 80 & 1059 & 1408 \\
\hline 100 & 1027 & 1444 \\
\hline 120 & 1091 & 1466 \\
\hline 140 & 1085 & 1468 \\
\hline 160 & 1100 & 1446 \\
\hline 180 & 1139 & 1473 \\
\hline 200 & 1160 & 1472 \\
\hline 220 & 1121 & 1435 \\
\hline
\end{tabular}

Fig 2 is showing graphical representation of RN-LEACH and Hybrid PSO-GA technique, $\mathrm{X}$-axis is representing the number of nodes and Y-axis is representing rounds. It has been clearly shown that in every round tenth node dies later in hybrid PSOGA than RN-LEACH.

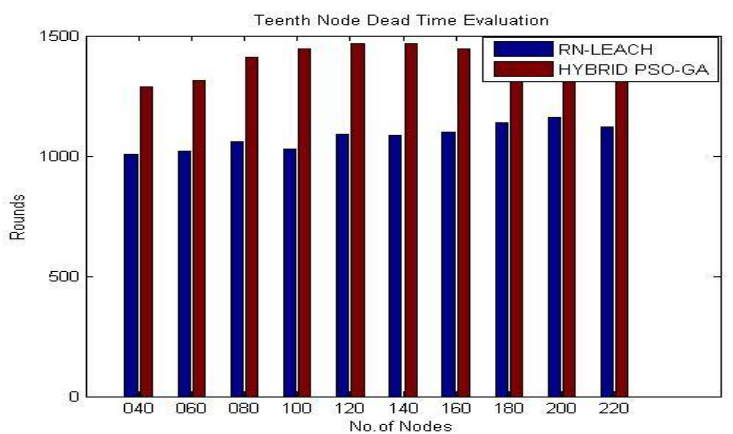

Figure 2. Graph when tenth node dies in RN-LEACH and Hybrid PSO-GA.

\subsection{Comparison on the basis of Network Lifetime}

Lifetime of the network is conducted when all the nodes of the network becomes dead. A node is said to be dead if its energy becomes zero (0). Table 3 shows the comparison of $\mathrm{RN}$ LEACH and the Hybrid PSO-GA on the basis of network lifetime. Using Hybrid PSO-GA the lifetime of the network is better than the RN-LEACH.

Table 3. Network Lifetime Analysis

\begin{tabular}{|c|c|c|}
\hline No. of nodes & RN-LEACH & PSO-GA \\
\hline 40 & 1439 & 1532 \\
\hline 60 & 1438 & 1539 \\
\hline 80 & 1435 & 1548 \\
\hline 100 & 1457 & 1584 \\
\hline 120 & 1452 & 1601 \\
\hline 140 & 1459 & 1560 \\
\hline 160 & 1458 & 1544 \\
\hline 180 & 1454 & 1566 \\
\hline 200 & 1449 & 1594 \\
\hline 220 & 1458 & 1587 \\
\hline
\end{tabular}

Fig 3. is showing graphical representation of RN-LEACH and Hybrid PSO-GA technique by taking different number of nodes with respect to rounds. $\mathrm{X}$-axis is representing the number of nodes and $\mathrm{Y}$-axis is representing rounds. It has been clearly shown that in every round lifetime of network is better in hybrid PSO-GA than RN-LEACH.

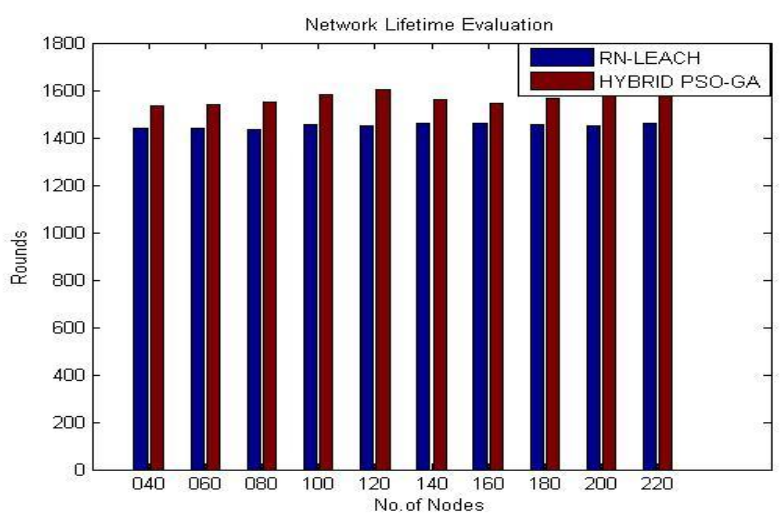

Figure 3. Graph shows the lifetime of the network in RNLEACH and Hybrid PSO-GA. 


\subsection{Comparison on the basis of Packet Delivery Ratio}

Packet delivery ratio represents the number of packets that are sent successfully to the base station Table 4 shows the comparison of RN-LEACH and the Hybrid PSO-GA on the basis of number of packets that are sent successfully to the base station. In the table it is clearly shown that more number of packets are sent by using hybrid PSO-GA technique than the RN-LEACH.

Table 4. Packet Delivery Ratio Analysis

\begin{tabular}{|c|c|c|}
\hline No. of nodes & RN-LEACH & PSO-GA \\
\hline 40 & 62.3782 & 69.1455 \\
\hline 60 & 63.9535 & 72.5918 \\
\hline 80 & 65.8271 & 74.3384 \\
\hline 100 & 69.2233 & 75.0229 \\
\hline 120 & 69.3306 & 75.8484 \\
\hline 140 & 69.5442 & 75.5035 \\
\hline 160 & 70.2726 & 75.0230 \\
\hline 180 & 70.4137 & 75.6475 \\
\hline 200 & 70.3194 & 77.2060 \\
\hline 220 & 70.4159 & 76.4136 \\
\hline
\end{tabular}

Fig 4 shows the comparison graph in which $\mathrm{x}$-axis reprsenting the number of nodes and y-axis representing the number of packets.

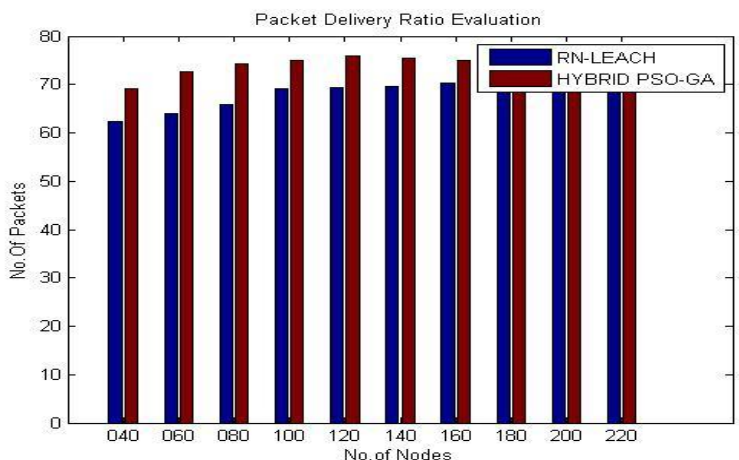

Figure 4 Comparison of RN-LEACH and Hybrid PSO-GA for Packet Delivery ratio

\subsection{Comparison on the basis Of remaining Energy}

Table 5 shows the comparison of RN-LEACH and the Hybrid PSO-GA on the basis of remaining energy left in the nodes after sending the data. In case of RN-LEACH more energy of nodes is consumed to send the data than Hybrid PSO-GA because PSO-GA provides the shortest path between cluster heads to base station. So less energy is consumed by each node to send the data.

Table 5. Average Remaining Energy Analysis

\begin{tabular}{|c|c|c|}
\hline No. of nodes & RN-LEACH & PSO-GA \\
\hline 40 & 0.0626 & 0.0708 \\
\hline 60 & 0.0621 & 0.0726 \\
\hline 80 & 0.0636 & 0.0735 \\
\hline
\end{tabular}

\begin{tabular}{|l|l|l|}
\hline 100 & 0.0643 & 0.0744 \\
\hline 120 & 0.0645 & 0.0749 \\
\hline 140 & 0.0647 & 0.0747 \\
\hline 160 & 0.0655 & 0.0749 \\
\hline 180 & 0.0665 & 0.0747 \\
\hline 200 & 0.0668 & 0.0753 \\
\hline 220 & 0.0662 & 0.0746 \\
\hline
\end{tabular}

Fig 5 is showing graphical representation of RN-LEACH and Hybrid PSO-GA technique. $\mathrm{X}$-axis is representing the number of nodes and Y-axis is representing rounds. It has been clearly shown that in every round remaining energy is better in hybrid PSO-GA than RN-LEACH.

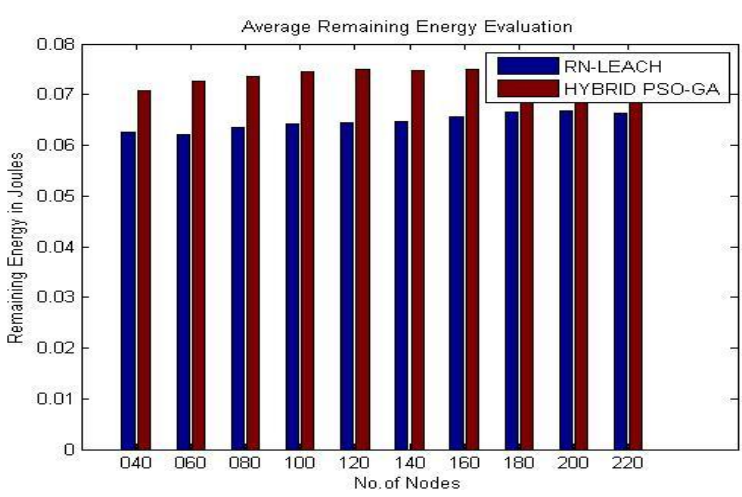

Figure 5. Comparison of RN-LEACH and Hybrid PSO-GA on the basis of Average Remaining Energy.

\section{CONCLUSION}

In this paper a new protocol is proposed a rendezvous nodes based LEACH protocol with the hybrid PSO-GA based routing. The proposed technique has improved the path selection between the sink and cluster heads to reduce the energy consumption of cluster heads in an optimistic manner. Due to non-availability of the actual environment, the simulation is considered for experimental purpose. The simulation is designed and implemented in the MATLAB tool using data analysis toolbox. Experimental results have shown that the proposed technique provides the better results on the basis of various parameters. This work has not considered the effect of 3D environment of sensor networks, therefore in near future the proposed protocol will be improved further for $3 \mathrm{D}$ WSNs.

\section{REFERENCES}

[1] A. Kumar, V. Kumar and N. Chand, "Energy Efficient Clustering and Cluster Head Rotation Scheme for Wireless Sensor Networks", (IJACSA) International Journal of Advanced Computer Science and Applications 3, no. 52011.

[2] G. Anastasi, M. Conti, M. Di Francesco and A. Passarella "Energy conservation in wireless sensor networks: A survey", Ad hoc networks7, no. 3,pp. 537-568, 2009.

[3] Ian F. Akyildiz, W. Su, Y. Sankarasubramaniam and E. Cayirci, "A survey on sensor networks", Communications magazine, IEEE 40, no. 8 , pp. 102-114, 2002.

[4] S.K. Singh, M. P. Singh and D. K. Singh,"Routing protocols in wireless sensor networks-A survey", 
International Journal of Computer Science \& Engineering Survey (IJCSES) Vol 1, pp. 63-83,2010.

[5] Al-Karaki, N. Jamal, and A. E. Kamal, "Routing techniques in wireless sensor networks: a survey", Wireless communications, IEEE 11, no. 6 ,pp .628,2004 .

[6] V. Potdar, A. Sharif, and E. Chang. "Wireless sensor networks: A survey." In Advanced Information Networking and Applications Workshops, 2009. WAINA'09. International Conference on, pp. 636-641. IEEE, 2009.

[7] Mao Ye, Chengfa Li, Guihai Chen, and Jie Wu. "EECS: an energy efficient clustering scheme in wireless sensor networks." In Performance, Computing, and Communications Conference, 2005. IPCCC 2005. 24th IEEE International, pp. 535-540. IEEE, 2005.

[8] Chunyao Fu, Zhifang Jiang, W. E. I. Wei, and Ang WEI "An Energy Balanced Algorithm of LEACH Protocol in WSN." IJCSI International Journal of Computer Science Issues 10, no. 1 , pp. 354-359. 2013.

[9] W. R. Heinzelman, A. Chandrakasan, and H. Balakrishnan. "Energy-efficient communication protocol for wireless microsensor networks." In System sciences, 2000. Proceedings of the 33rd annual Hawaii international conference on, pp. 10-pp. IEEE, 2000.

[10] M. M. Salim,, H. A. Elsayed, and S. H. El Ramly. "PRLEACH: Approach for balancing energy dissipation of LEACH protocol for wireless sensor networks." In Radio Science Conference (NRSC), 2014 31st National, pp. 252259. IEEE, 2014.

[11] G. Xing,, T. Wang, W. Jia, and M. Li. "Rendezvous design algorithms for wireless sensor networks with a mobile base station." InProceedings of the 9th ACM international symposium on Mobile ad hoc networking and computing, pp. 231-240. ACM, 2008.

[12] S. Mottaghi,, and M. R. Zahabi. "Optimizing LEACH clustering algorithm with mobile sink and rendezvous nodes." AEU-International Journal of Electronics and Communications 69, no. 2. pp.507-514, 2015.
[13] G. Xing,, T. Wang, Z. Xie, and W. Jia, "Rendezvous planning in mobility-assisted wireless sensor networks." In Real-Time Systems Symposium, 2007. RTSS 2007. 28th IEEE International, pp. 311-320. IEEE, 2007.

[14] Ian F. Akyildiz, W. Su, Y. Sankarasubramaniam, and E. Cayirci, "Wireless sensor networks: a survey." Computer networks 38, no. 4, pp. 393-422. 2002.

[15] K. Akkaya, and M. Younis. "A survey on routing protocols for wireless sensor networks." Ad hoc networks 3, no. 3, pp. 325-349, 2005.

[16] L. T. Nguyen, X. Defago, R. Beuran, and Y. Shinoda. "An energy efficient routing scheme for mobile wireless sensor networks", InWireless Communication Systems. 2008. ISWCS'08. IEEE International Symposium on, pp. 568-572. IEEE, 2008

[17] J. Singh, B. P. Singh, and S. Shaw, "A new LEACHbased routing protocol for energy optimization in wireless sensor network", In Computer and Communication Technology (ICCCT), 2014 International Conference on, pp. 181-186. IEEE, 2014

[18] D. Amine, B. Nassreddine, and K. Bouabdellah. "Energy efficient and safe weighted clustering algorithm for mobile wireless sensor networks." Procedia Computer Science 34, pp. 63-70, 2014

[19] S. Bayrakl1,, and S. Z. Erdogan, "Genetic algorithm based energy efficient clusters (GABEEC) in wireless sensor networks." Procedia Computer Science 10 , pp. 247-254, 2012

[20] $\mathrm{Hu} \mathrm{Yu}$, and Wang Xiaohui, "PSO-based Energy-balanced Double Cluster-heads Clustering Routing for wireless sensor networks." Procedia Engineering15, pp. 30733077, 2011.

[21] You-Chiun Wang,, Fang-Jing Wu, and Yu-Chee Tseng, "Mobility management algorithms and applications for mobile sensor networks." Wireless Communications and Mobile Computing 12, no. 1, pp. 7-21, 2012 